\title{
Osteologia craniana comparada de Caracara cheriway (Jacquin, 1784) e Caracara plancus (Miller, 1777) (AVES: FALCONIDAE)
}

\author{
Cranial osteology compared to Caracara cheriway (Jacquin, 1784) and Caracara plancus \\ (Miller, 1777) (AVES: FALCONIDAE)
}

\author{
Anderson Guzzi ${ }^{1 *}$, Jussyleny Maria do Nascimento Castro², Raimundo Nonato Cornelio \\ Martins Junior ${ }^{2}$, Reginaldo José Donatelli³, Leonardo Moura dos Santos Soares ${ }^{4}$ e \\ Guilherme José Bolzani de Campos Ferreira ${ }^{5}$
}

\author{
${ }^{1}$ Doutor, Departamento de Ciências do Mar, Universidade Federal do Piauí, Parnaíba, Brasil \\ ${ }^{2}$ Graduado, Departamento de Ciências do Mar, Universidade Federal do Piauí, Parnaíba, Brasil \\ ${ }^{3}$ Doutor, Faculdade de Ciências, Universidade Estadual Paulista, Bauru, Brasil \\ ${ }^{4}$ Doutor, Museu Paraense Emílio Goeldi (MPEG), Belém, Brasil \\ ${ }^{5}$ Doutor, Curso de Medicina Veterinária, Universidade Federal do Piauí, Bom Jesus, Brasil
}

\begin{abstract}
Resumo
O gênero Caracara (Aves: Falconidae) é composto por C. cheriway (caracará-do-norte) e C. plancus (caracará). O presente trabalho teve como objetivo descrever de forma detalhada e comparada a osteologia craniana dessas duas espécies, identificando caracteres para futuros trabalhos sistemáticos. Os caracteres comuns das espécies de Caracara são: a projeção do rostro paraesfenoidal alcança $50 \%$ da distância do côndilo occipital ao pterigóide; maxila superior tem cerca de 4/9 do comprimento total do crânio; a parte sinfisial da mandíbula ocupa cerca de 1/7 do comprimento total; a parte proximal do lacrimal tem cerca de 1/4 da largura da parte distal. Os caracteres exclusivos de C. cheriway são: a largura interorbital é cerca de 1/2 daquela da região parietal; há uma proeminência do osso frontal; a parte proximal do lacrimal atinge 1/5 da distância do arco da órbita até a barra jugal; a distância entre as partes distais dos dois lacrimais atinge 5/6 da largura parietal; a razão entre o diâmetro interorbital e a região parietal varia de duas vezes; o processo zigomático ocupa $40 \%$ da distância entre a sua origem no crânio e a barra jugal. Os caracteres exclusivos de C. plancus são: a largura interorbital é cerca de 4/7 da largura parietal; há uma concavidade rostral mediana; a parte proximal do lacrimal tem cerca de $1 / 4$ da largura da parte distal; a parte proximal do lacrimal atinge 1/7 da distância do arco da órbita até a barra jugal; a distância entre as partes distais dos dois lacrimais atinge 6/7 da largura parietal; a razão entre o diâmetro interorbital e a região parietal varia de 1,75 vezes; o processo zigomático ocupa $35 \%$ da distância entre a sua origem no crânio e a barra jugal. Palavras-chave: Anatomia. Caracara cheriway. Caracara plancus. Falconidae. Osteologia craniana
\end{abstract}

\begin{abstract}
The Caracara genus (Aves: Falconidae) is composed by species C. cheriway, ("Crested Caracara"), and C. plancus (Southern Caracara). This study aimed to describe in detail and compared the cranial osteology of C. cheriway and C. plancus, identifying osteological cranial characters for a future systematic work. The two common characters of Caracara species are: the projection of rostro parasphenoid reaches $50 \%$ of the distance from the occipital condyle to the pterygoid; the upper maxilla has about 4/9 of the total length of theskull, the symphysial portion of mandible occupies about 1/7 of its total length, the proximal portion of lacrimal bone ends in a rounded shape and has about 1/4 of the width of the distal portion. The unique characters of $C$. cheriway are: the interorbital width is about 1/2 of the parietal region, shows a frontal bone prominence; presence of lacrimal process of frontal bone; the proximal portion of the lacrimal bone reaches $1 / 5$ of the distance from the orbital arc to the jugal bone; the distance between the distal portions of two lacrimal bones reach 5/6 of the parietal width, the ratio between the interorbital diameter and parietal region ranges from 2 times; the zygomatic process occupies about $40 \%$ of the distance between its origin in the skull and jugal bone. The unique characters of C. plancus are: the interorbital width is approximately 4/7 of the parietal width; show a rostral medial concavity; the proximal portion of the lacrimal bone is about 1/4 of the width of the distal; the proximal portion of the lacrimal bone reaches 1/7 of the distance from the orbital arc to the jugal bone; the distance between the distal portions of two lacrimal bones reaches 6/7 of the width parietal one; the ratio between the interorbital diameter and parietal region varies 1, 75 times, the zygomatic process occupies about $35 \%$ of the distance between its origin in the skull and jugal bone.

Keywords: Anatomy. Caracara cheriway. Caracara plancus. Falconidae. Cranial Osteology
\end{abstract}




\section{Introdução}

$\mathrm{D}$ entre os vertebrados recentes, as Aves constituem um grupo dos mais bem estudados e conhecidos talvez pela facilidade de observação no campo, devido principalmente à sua vocalização e coloração (DONATELLI, 1992). Os Falconidae constituem uma família de aves pertencentes à ordem Falconiformes e incluem cerca de 68 espécies de aves de rapina (GILL; DONSKER, 2014), das quais 20 ocorrem no Brasil, distribuídas em 07 gêneros (CBRO, 2014).

Griffiths (1999), utilizando caracteres anatômicos combinados a dados moleculares, considera a família Falconidae monofilética, dividindo-a em duas subfamílias, Herpetotherinae e Falconinae. Segundo esse autor, Herpetotherinae abriga os gêneros Micrastur e Herpetotheres, ao passo que Falconinae é subdividida em duas tribos: Falconini (engloba os gêneros Falco, Spiziapterix, Neohierax, Microhierax e Polihierax) e Caracarini (compreende os gêneros Caracara, Ibycter, Milvago, Phalcoboenus e Daptrius). Fuchs et al. (2011), utilizando somente dados moleculares, corrobora igualmente os resultados obtidos por Griffiths (1999).

Os Caracarini (caracaras) compreendem seis gêneros e 12 espécies endêmicas do Novo Mundo, sendo a mais diversa subfamília de Falconidae em morfologia e comportamento, com distribuição na América Central/ América do Sul (11 espécies endêmicas) (FUCHS et al., 2012), compondo um clado que inclui todas as grandes espécies onívoras (BROWN; AMADON, 1968; STRESEMANN; AMADON, 1979). Spiziapteryx circumcincta foi o primeiro taxon a se ramificar dentro dos Polyborinae, em torno de $14 \mathrm{Ma}$. Todas as outras espécies tradicionalmente referidas como caracaras formam um grupo monofilético, sendo Caracara o primeiro gênero a divergir em aproximadamente 6,7 Ma, sugerindo que o comportamento alimentar generalista e necrófago em falcões apareceu entre 14 e 6,7 Ma (FUCHS et al., 2012).

A taxonomia das espécies do gênero Caracara foi por muito tempo discutida e alvo de controvérsias. Rigdway (1876) os tratou como três táxons distintos: C. plancus [descrito por Miller (1777)], correspondendo aos espécimes que se distribuem ao sul da América do Sul; $C$. cheriway [descrito por Jacquin (1784)], correspondendo aos espécimes que se distribuem ao norte da América do Sul até o sul da América do Norte (Colômbia, Equador, norte do Peru, Venezuela, Trinidad, Guianas) e ao norte do Brasil, ao longo do baixo rio Amazonas (DOVE; BANKS, 1999) e Rigdway (1876) descreve C. lutosus com distribuição na Ilha de Guadalupe no México.

Posteriormente, Hellmayir e Conover (1949) foram os primeiros a tratar os táxons continentais (C. plancus e C. cheriway) como coespecíficos. Os principais caracteres que os diferenciavam (coloração e comprimento total) foram tratados como diferenciação clinal. Em síntese, alguns autores os consideravam coespecíficos (HELLMAYIR; CONOVER, 1949; WETMORE, 1965; BROWN;
AMADON, 1968; BLAKE, 1977; STRESEMANN; AMADON, 1979; SIBLEY; MONROE, 1990) e outros autores os consideravam como táxons distintos (RIGDWAY, 1876; FRIEDMANN et al., 1950).

Dove e Banks (1999) analisaram os três táxons do gênero Caracara levando em consideração a distribuição geográfica, caracteres morfométricos e plumagem de vários exemplares. Os autores elevaram a subespécie Caracara plancus cheriway à condição de espécie independente (válida) tendo como principal barreira geográfica ao fluxo gênico o rio Amazonas, o mesmo padrão observado por Vuilleumier (1970) ao analisar seis caracteres morfométricos: tamanho, proporção, padrão e cor da plumagem, pele facial nua, forma da narina e preferência de habitat; e por fim, Fuchs et al. (2012) considera o gênero Caracara monofilético e suas duas espécies (C. plancus e $C$. cheriway) válidas, comentando que elas diferem entre si somente em $0,5 \%$ quanto aos genes mitocondriais.

O caracará-do-norte Caracara cheriway tem entre 54 e $59 \mathrm{~cm}$ de comprimento total e é muito semelhante ao caracará (Caracara plancus) (DOVE; BANKS, 1999). Alimenta-se principalmente de carniça, complementada de pequenos vertebrados (peixes, tartarugas e filhotes de aves) e invertebrados (MORRISON et al., 2008). Esta espécie caça suas presas ao caminhar no solo, principalmente em áreas agrícolas e ao longo de estradas onde procura animais atropelados. Sobrevoa queimadas para predar animais espantados pelo fogo ou animais mortos (GLOBAL RAPTORS, 2010). Os ninhos de Caracara cheriway são encontrados em arbustos altos, espinhosos, densamente ramificados e com cobertura vegetal ainda viva, de modo a oferecer uma maior ocultação em áreas abertas (ACTKINSON et al., 2007), utilizando o ninho por várias gerações, e os juvenis formam grupos e utilizam a área de forrageamento de seus pais o máximo de tempo possível, até delimitarem seus próprios territórios (DWYER et al., 2013).

Caracara plancus é uma das espécies mais comuns e populares do Brasil. Conhecido também como carrancho (sul do Brasil), carcará e caracaraí (na Ilha de Marajó) além de gavião-de-queimada (SICK, 1997). Medindo em média $56 \mathrm{~cm}$ de comprimento total e $123 \mathrm{~cm}$ de envergadura, é facilmente reconhecível quando pousado por possuir uma espécie de solidéu preto sobre a cabeça e um bico adunco e alto, que se assemelha à lâmina de um cutelo; a face é vermelha. É recoberto de preto na parte superior e possui peito marrom claro com riscas pretas, patas compridas de cor amarela; em voo, assemelha-se a um urubu, mas é reconhecível por duas manchas de cor clara na extremidade das asas (SICK, 1997). A alimentação de C. plancus inclui carniça, vários grupos de artrópodes, anfíbios, répteis, aves e até mesmo frutos (DEL HOYO et al., 1994; FERGUSON-LEES; CHRISTIE, 2001); saqueia ninhos de garças e de outros gaviões. Seu ninho é construído com ramos sobre as árvores ou diretamente no solo. São colocados 2 ou 3 ovos, raramente 
4, incubados pelo casal durante 28 dias. Geralmente os pais criam um só filhote por ninhada (HOFLING, 1993; SICK, 1997).

Este trabalho descreve a osteologia craniana de Caracara cheriway comparado a C. plancus, pois os trabalhos encontrados em literatura são em sua maioria de comportamento alimentar (ZOTTA, 1940; HERTEL, 1995; MONTALVO et al., 2011; TRAVAINI et al., 2001; GALETTI; GUIMARÃES, 2004; MORRISON et al., 2007; SAZIMA, 2007), comportamento reprodutivo (MORRISON et al., 2003; ACTKINSON et al., 2007; SKORUPPA; LEE, 2008; DWYER et al., 2013), ecologia (BAUMGARTEN, 1998), histologia (NEGRO et al., 2006), parasitologia (VITALIANO et al., 2010) e poucos de anatomia (FRANZO et al., 2007; SUSTAITA, 2008; SUSTAITA; HERTEL, 2010 e MOSTO et al., 2013).

A anatomia foi um tema bastante explorado no final do século XIX, e embora tenha contribuído para a compreensão dos grandes grupos de aves, os estudos anatômicos tradicionais foram pouco elucidativos sistematicamente em níveis categóricos inferiores, pois as comparações eram feitas frequentemente entre táxons filogeneticamente distantes entre si (POSSO ; DONATELLI, 2007) que compartilham aspectos de sua história de vida, provocando interpretações intuitivas acerca da convergência dos caracteres morfológicos, sobressaltando a dependência da anatomia para estudos sistemáticos (LIVEZEY; ZUSI, 2007). Mas estudos de filogenia dentro de gêneros e famílias de aves proveram uma nova oportunidade para a análise da anatomia num contexto filogenético (LIVEZEY, 1986 e 1991), e o crânio de aves pôde representar uma parte principal em análises filogenéticas, e evidências anatômicas para seus estados foram levantadas com sucesso (ZUSI, 1993).

Posto isso, o objetivo deste trabalho é descrever de forma detalhada e comparada a osteologia craniana de C. cheriway e C. plancus, buscando levantar caracteres que subsidiem futuros estudos sistemáticos.

\section{Materiais e Métodos}

O estudo foi realizado com base na descrição dos ossos do crânio de cinco espécimes de $C$ cheriway [USNM 322388 (fêmea, cativeiro); USNM 553230 (sexo indeterminado, Andes, Chile); USNM 431612 (macho, Cativeiro); USNM 19671 (macho, Fla, Kissimmee); USNM 321805 (macho, cativeiro), e cinco espécimes de C. plancus [USNM 490931 (fêmea, Ilha dos Estados, Argentina); USNM 555500 (fêmea, cativeiro); USNM 345780 (macho, Cáceres, Brasil); USNM 428041 (macho, cativeiro); USNM 614583 (macho, Entre Rio, Argentina)], que fazem parte da coleção osteológica da Divisão de Aves do Museu de História Natural Smithsonian Institution (USNM), Washington, DC, EUA.

A descrição da osteologia craniana de Caracara plancus e C. cheriway foi comparativa e ilustrada por pranchas em vista dorsal, ventral, caudal e lateral, acompanhadas por legendas para facilitar a observação das estruturas.

Considerando que a maioria dos ossos cranianos é par e que existe simetria bilateral, a descrição osteológica se deu no singular. A nomenclatura para a descrição da osteologia e das demais estruturas associadas seguiu a Nomina Anatomica Avium (BAUMEL; WITMER, 1993). A nomenclatura e a classificação seguiram o Comitê Brasileiro de Registros Ornitológicos (CBRO, 2014).

\section{Resultados}

\subsection{Ossa cranii}

A região frontal de Caracara cheriway (Os frontale F, Fig. 1) ocupa grande parte do teto craniano, cerca de metade do comprimento total da caixa craniana, apresentando uma depressão frontal (DF - Figura 1). A largura interorbital (1, Figuras 1 e 8 ) é cerca de $1 / 2$ da região parietal em Caracara cheriway, ao passo que em C. plancus é cerca de $4 / 7$ da largura parietal (2, Figuras 1 e 8). A região frontal articula-se com a região nasal rostralmente por meio da zona flexória craniofacial (ZFC - Figuras 1 e 8); a zona está descrita como articulação sinovial do tipo gínglimo, contudo a mesma se apresenta parcialmente fundida medialmente em C. cheriway. Em C. cheriway, essa região apresenta uma proeminência do frontal (PF, Fig. 1), ao passo que C. plancus há uma concavidade rostral mediana (CRM, Fig. 8). A região frontal continua-se caudalmente com a ampla região parietal e laterocaudalmente se estende com o longo processo pós-orbital em ambas as espécies. A comissura rostral de os lacrimale tem cerca de $1 / 4$ da largura da borda caudal em C. cheriway e, em C. plancus, terminando de forma arredondada em ambas as espécies, não formando uma comissura e atingindo $1 / 5$ da distância do arco da órbita até a barra jugal (AJ, Figuras 1, 2 e 3) em C. cheriway e 1/7 em C. plancus. A distância entre as partes caudais dos dois ossos lacrimais atinge 5/6 da largura parietal em C. cheriway, enquanto que em C. plancus essa distância atinge 6/7 da largura parietal.

Em ambas as espécies, a ampla região parietal (Os parietale - P, Figuras 1, 2 e 8) limita-se lateroventralmente com a região esquamosal por meio da conspícua crista temporal superior (CrTS - Figuras 2, 9 e 11), laterocaudalmente contata-se com a região occipital por meio da discreta crista nucal transversa (crista nuchalis transversa - CrNT, Figuras 4 e 11); em sua região mediana caudal continua-se com a região supra-occipital. A razão encontrada entre o diâmetro interorbital e a região parietal varia de 2 vezes para C. cheriway e 1,75 vezes para $C$. plancus.

A região esquamosal (Os squamosum) é delimitada laterodorsalmente pela crista temporal superior (CrTS - Figuras 2, 9 e 11), que ocupa grande parte da porção lateral do crânio, caudalmente ao processo pós-orbital; 

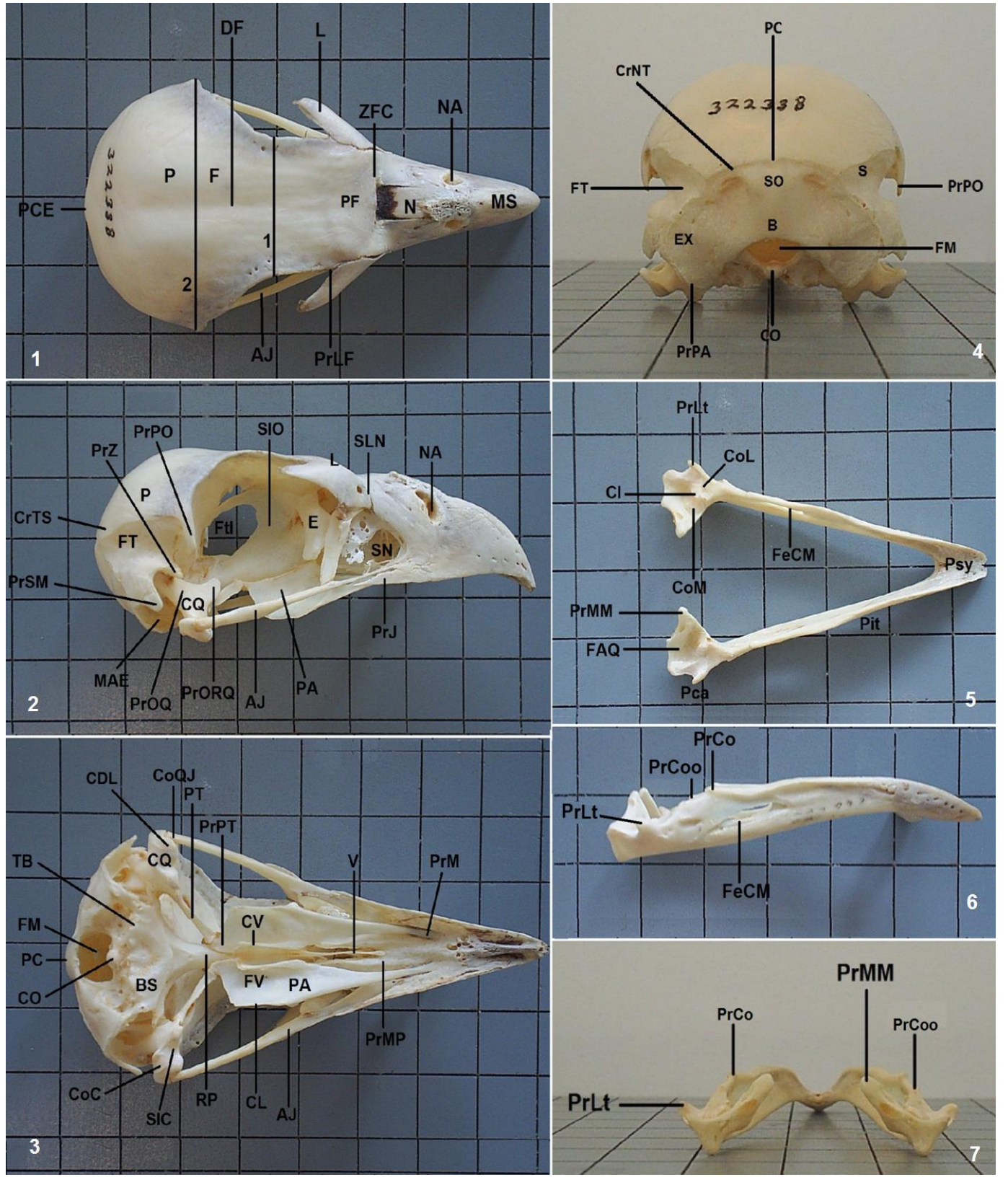

Figuras 1 a 7. Vistas do crânio e mandíbula de Caracara cheriway. Notar que o quadriculado da figura tem um centímetro de lado. 1: Vista dorsal do crânio. 2: Vista Lateral do crânio. 3: Vista ventral do crânio. 4: Vista caudal do crânio. 5: Vista dorsal da mandíbula. 6: Vista lateral da mandíbula. 7: Vista caudal dos ramos mandibulares. Legenda: 1: largura interorbital; 2: largura parietal; AJ: arco jugal; B: basioccipital; BS: região basisfenóide; CDL: côndilo lateral; CI: crista intercotilar; CL: crista lateral palatina; CO: côndilo occipital; CoC: côndilo caudal; CoL: cótila lateral; CoM: cótila medial; CQ: corpo do osso quardrado; CrNT: crista nuchalis transversa; CrTS: crista temporal superior; CV: crista ventral palatina; DF: depressão frontal; E: ectetmóide; EX: exoccipital; F: frontal; FAQ: fossa articular quadrática; FeCM: fenestra caudal da mandíbula; FM: forâmen magno; FT: fossa temporal; FV: fossa ventral palatina; L: lacrimal; MAE: meato acústico externo; MS: maxila superior; NA: abertura nasal; P: parietal; PC: proeminência cerebelar; Pca: parte caudal; PF: proeminência frontal; Pit: parte intermediária; PrCo; o processo coronóide 1; PrCoo: processo coronóide 2; PrJ: processo jugal; PrLF: processo lacrimal do frontal; PrLt: processo lateral da mandíbula; PrM: processo maxilar do palatino; PrMM: processo medial da mandíbula; PrMP: processo maxilopalatino; PrOQ: processo ótico do quadrado; PrORQ: processo orbital do quadrado; PrPA processo paraoccipital; PrPO: processo pós-orbital; PrPT: processo transpalatino; PrSM: processo suprameático; PrZ: processo zigomático; Psy: parte sinfisial da mandíbula; PT: pterigoide; RP: rostro paraesfenoidal; SIO: septo interorbital; SLN: sutura lacrimonasal; SO: supra-occipital; TB: tubérculo basilar; V: vômer; ZFC: zona flexória craniofacial. 
ventrocaudalmente pelo pronunciado processo suprameático (Proc. suprameaticus - PrSM, Figuras 2 e 9) presente nas espécies estudadas e pelo meato acústico externo (Meatus acusticus externus - MAE, Figuras 2 e 9). A fossa temporal (Fossa temporalis - FT, Figuras 2, 4, 9 e 11) é mais profunda na parte proximal aos processos pós-orbital e zigomático e mais rasa na parte distal, sendo mais longa rostrocaudalmente que larga, profunda em ambas as espécies. A fossa temporal é delimitada dorsalmente pela crista temporal superior (CrTS - Figuras 2,9 e 11) e ventralmente pela crista nucal transversa (CrNT - Figuras 4 e 11).

A região esquamosal projeta-se rostralmente e forma o processo zigomático (Proc. zigomaticus - PrZ, Figuras 2 e 9). Tal processo apresenta-se longo e alargado em sua base e apresenta-se em forma de um triângulo característico, levemente torcido sobre si mesmo, ocupando cerca de $40 \%$ da distância entre a sua origem no crânio e a barra jugal em C. cheriway, em 35\% em C. plancus. O processo zigomático possui as faces dorsal, ventral, lateral e medial bem delimitadas, evidenciando os locais de origem do músculoadutor mandibular externo $(M$. adductor mandibulae externus) e de duas de suas porções (pars temporalis, pars medialis e pars profunda).

A região esquamosal delimita-se rostromedialmente com a região laterosfenóide e rostrodorsalmente com a região frontal por meio do processo pós-orbital (Proc. postorbitalis - PrPO, Figuras 2, 4, 9 e 11). Tal processo é longo e robusto em ambas as espécies, com base larga e levemente torcido sobre si mesmo.

A região occipital compreende os ossos supra-occipital (Os supraoccipitale-SO, Figuras 4 e 11), exoccipital (Os exoccipitale - EX, Figuras 4 e 11) e basoccipital (Os basioccipitale - B, Figuras 4 e 11) fusionados. Limita-se dorsolateralmente com a região esquamosal por meio do meato acústico externo e da crista nucal transversa. A região do osso exoccipital delimita-se com a ala timpânica (Cavum tympanicum-AT, Fig. 10) por meio do pronunciado processo paraoccipital (Proc. paraoccipitalis-PrPA, Figuras 4 e 11). O seu limite com a região basioccipital é marcado pela presença de foramens por onde passam nervos (Foramen n. glossopharingealis, n.vagi e canalis $n$. hypoglossi) e de canais por onde passam vasos (Ostium canalis carotici e ostium canalis ophthalmici externi) e pelo processo condilar. A região do osso basioccipital abriga o conspícuo côndilo occipital (Condylus occipitalis-CO, Figuras 3, 4 e 10) e a profunda fossa subcondilar (FS, Figura 10) é circundada por dois tubérculos basilares bem desenvolvidos (Tuberculum basilare- TB, Figuras 3 e 10). Caudomedialmente observa-se o largo forâmen magno (Foramen magno - FM, Figuras 3, 4, 10 e 11). A proeminência cerebelar da região do osso supra-occipital é saliente e afilada (PC, Figuras 3,4 e 10) tanto em $C$. cheriway, quanto em C. plancus.

A região basioccipital continua-se rostralmente com a região basisfenóide por meio do tubérculo basilar. A região basisfenóide (Os basisphenoidale - BS, Figuras 3 e
10) é alargada em sua base, estreitando-se rostralmente para se fundir com a região paraesfenóide, essas duas regiões somadas à região basioccipital formam a maior parte do assoalho do crânio (Cranium).

Na região do osso paraesfenóide (Os parasphenoidale), destaca-se a projeção do rostro paraesfenoidal (Rostrum parasphenoidale - RP, Figuras 3 e 10), que alcança 50\% da distância do côndilo occipital e o pes pterygoidei em ambas as espécies, articulando-se rostralmente com o palatino e, rostrolateralmente, com o pterigóide; funde-se dorsalmente ao septo interorbital; rostralmente contata-se ainda com o osso vômer por meio da facies articularis vomeralis.

\subsubsection{Orbita}

O osso ectetmóide (Os ectethmoidale - E, Figuras 2 e 9) apresenta-se fundido ao osso lacrimal em toda sua extensão, apresentando a forma da letra " $\mathrm{D}$ " deitada, apresentando um processo pronunciado nas duas espécies. Em sua face nasal, rostrolateralmente funde-se com a face laterocaudal da região do osso frontal e lacrimal (L, Figuras 1, 2 e 8). Entre esses dois ossos há uma pequena expansão lateral do frontal, denominada processo lacrimal do frontal (Proc. Lacrimalis) (PrLF, Fig. 1), que é presente em $C$. cheriway e ausente em $C$. plancus. Rostromedialmente funde-se com a região rostral do septo interorbital. O osso ectetmóide é muito bem desenvolvido e de fácil delimitação na órbita em ambas as espécies analisadas.

O septo interorbital (Septum interorbitale -SIO, Figuras 2 e 9) delimita-se dorsalmente com a região do osso frontal por meio do sulco olfatório (Sulcus olfactorii-SNO, Figura 9) e do conspícuo forâmen orbitonasal medial (Foramen orbitonasale mediale- Fom, Fig. 9). Limita-se rostrolateralmente com o osso ectetmóide e ventrolateralmente contata-se ao rostro paraesfenóide e ao Processo palatino; e ventrolateralmente ao osso pterigóide.

A região laterosfenóide localiza-se na porção caudal da órbita; estende-se da porção caudal do septo interorbital até a face lateral da região esquamosal e do processo pós-orbital. Em sua face temporal, porção dorsolateral da região laterosfenóide, observa-se lateralmente o processo pós-orbital. Em sua facies cerebralis, medialmente disposta ao processo pós-orbital, destaca-se uma depressão característica da região laterosfenóide. Ventralmente a este tubérculo observa-se que o forâmen do nervo maxilomandibular é estreito (FoNM, Figura 9). Dorsalmente a este forâmen observa-se o forame do nervo oftálmico (Foramen n. ophthalmici), com calibre maior que o primeiro. Na parte medial da orbita, porção dorsomedial da região laterosfenóide, destaca-se o fontículo orbitocranial, que atinge também a porção caudal do septo interorbital (Septum interorbitale - SIO, Figuras 2 e 9). Na região caudal da órbita encontra-se o forame do nervo óptico (Forâmen nervo optici), bem desenvolvido. A região laterosfenóide é delimitada ventrolateralmente pelo processo zigomático da região 
esquamosal e medialmente delimita-se com o septo interorbital por meio do fontículo orbitocranial.

\subsection{Ossa faciei}

\subsubsection{Ossa Maxillae}

A maxila superior (MS - Figuras 1 e 8) é formada pela fusão dos ossos pré-maxilares (Os premaxillare), maxilares (Os maxillare) e nasais (Os nasale); tem cerca de 4/9 do comprimento total do crânio em $C$. cheriway e em C. plancus. As narinas (NA - Figuras 1, 2, 8 e 9) têm um formato circular e caracterizam-se como do tipo holorrina. O processo maxilar lateral do osso nasal é largo, curto e inclinado; as barras dorsais do processo maxilar do nasal, longo, afilam-se rostralmente e delimitam a porção dorsocaudal da narina. $\mathrm{O}$ nasal articula-se ao lacrimal através da sutura lacrimonasal (SLN - Figura 2). A zona flexória craniofacial ocupa somente a porção mediana entre as regiões frontal e nasal; lateralmente há uma nítida fusão entre essas regiões. A barra dorsal encontra-se fusionada à face rostrolateral da região do osso frontal, o mesmo ocorrendo com a barra lateral mais lateralmente.

\subsubsection{Palati}

A região do palato é formada pelos ossos palatinos (Os palatinum), pterigóides e vômer. O processo maxilar do palatino (Proc. maxillaris - PrM, Figuras 3 e 10) conecta a lamela caudolateral palatina à base da maxila superior. Este processo é longo e estreito em sua extensão nas duas espécies estudadas. A lâmina caudolateral palatina (Lamella caudolateralis) da pars lateralis é delimitada medialmente pela proeminente crista ventral palatina (Crista ventralis - CV, Figuras 3 e 10) da pars choanalis, pelo processo transpalatino (PrPT - Figuras 3 e 10) e, lateralmente, pela longa crista lateral palatina (Crista lateralis - CL, Figuras 3 e 10) em ambas as espécies; entre as cristas lateral e ventral do palatino observa-se uma profunda fossa ventral palatina (Fossa ventralis-FV, Figuras 3 e 10) presente em ambas as espécies. Assim, a fossa ventral palatina é curta, estreita, profunda e forma a maior parte da lamela caudolateral palatina nas espécies estudadas. As cristas ventrais palatinas são longas e proeminentes, e encontram-se na região de articulação com o paraesfenóide. A articulação com o pterigóide faz-se dorsolaterocaudalmente, por meio da pars pterygoidea palatina que apresenta o curto processo pterigóide do palatino nas duas espécies. As cristas mediais palatinas (Crista medialis-CrM, Figura 10) são proeminentes, terminando de forma afilada, situandose mais medialmente e delimitando a fossa coanal (FC, Figura 10). A pars choanalis é estreita e mais desenvolvida se comparada à pars lateralis descrita acima.

O osso vômer (V - Figuras 3 e 10) apresenta-se como uma lâmina longa e estreita nas duas espécies estudadas, achatada bilateralmente, pontiagudo rostralmente; articula-se caudalmente com a pars choanalis do palatino e com o rostro paraesfenóide por meio da facies articularis vomeralis.

O osso quadrado localiza-se em uma região central do crânio e interliga o palato, o crânio e a mandíbula, desempenhando um papel fundamental na cinética craniana. Apresenta um corpus ossi quadrati (CQ - Figuras 2, 3,9 e 10) central de onde partem três processos: orbital, ótico e mandibular.

O processo orbital do quadrado (Proc. orbitalis PrORQ, Figura 2) projeta-se rostromedialmente na órbita; apresenta-se curto e largo ambas as espécies estudadas, afilando-se tenuamente distalmente e terminando de forma arredondada. Assume claramente uma posição oblíqua na órbita.

O processo ótico do quadrado (Proc. oticus - PrOQ, Figuras 2 e 9) é longo e alargado nas espécies analisadas a partir do corpo quadrático. Articula-se com a caixa craniana em dois locais distintos: a) por meio do processo ótico externo (Capitulum squamosum) presente tanto em C. cheriway quanto em C. plancus, apoiado na face ventrocaudal do processo zigomático; e b) por meio do processo ótico interno (Capitulum oticum), presente nas espécies estudadas, que se articula com as cotylae quadraticae otici dos ossos proótico e opistótico.

O processo mandibular do quadrado apresenta quatro côndilos distintos: o côndilo medial (Condylus medialis), que é arredondado, e o côndilo lateral, (Condylus lateralis - CDL, Figuras 3 e 10) que é longo e mais robusto que o medial, articulando-se com a barra jugal lateralmente a partir de um processo dorsal; o côndilo caudal (Condylus caudalis - CoC, Fig. 3) é uma extensão do côndilo lateral, sendo fusionado a ele. O côndilo caudal é maior que o medial nas duas espécies, e o côndilo pterygoideus, localizado medialmente, articula-se com o pterigóide por meio de sua face articular quadrática.

O côndilo lateral do quadrado possui ainda a cótila quadradojugal (Cotyla quadratojugalis), que se articula com o côndilo quadraticus da barra jugal; trata-se de uma sutil depressão por onde se articula a barra jugal com o quadrado. A face medial do quadrado, particularmente aquela relativa ao corpus ossi quadrati, possui uma fossa característica, mais larga que profunda.

O pterigóide (Pterygoideum - PT, Figuras 3 e 10) tem uma expansão rostral que se alarga rostrodorsalmente; articula-se com o palatino dorsolaterocaudalmente por meio de uma estrutura denominada pes pterygoidei. De uma forma geral, o pterigóide apresenta-se como uma lâmina relativamente achatada e disposta obliquamente na órbita. Articula-se laterocaudalmente com o osso quadrado por meio da facies articularis quadratica do processo quadrático do pterigóide (Proc. quadraticus --PrQ, Figura 10).

\subsection{Ossa mandibulae}

A parte sinfisial da mandíbula (pars symphisialis mandibulae - Psy, Figuras 5 e 12) ocupa cerca de 1/7 do 


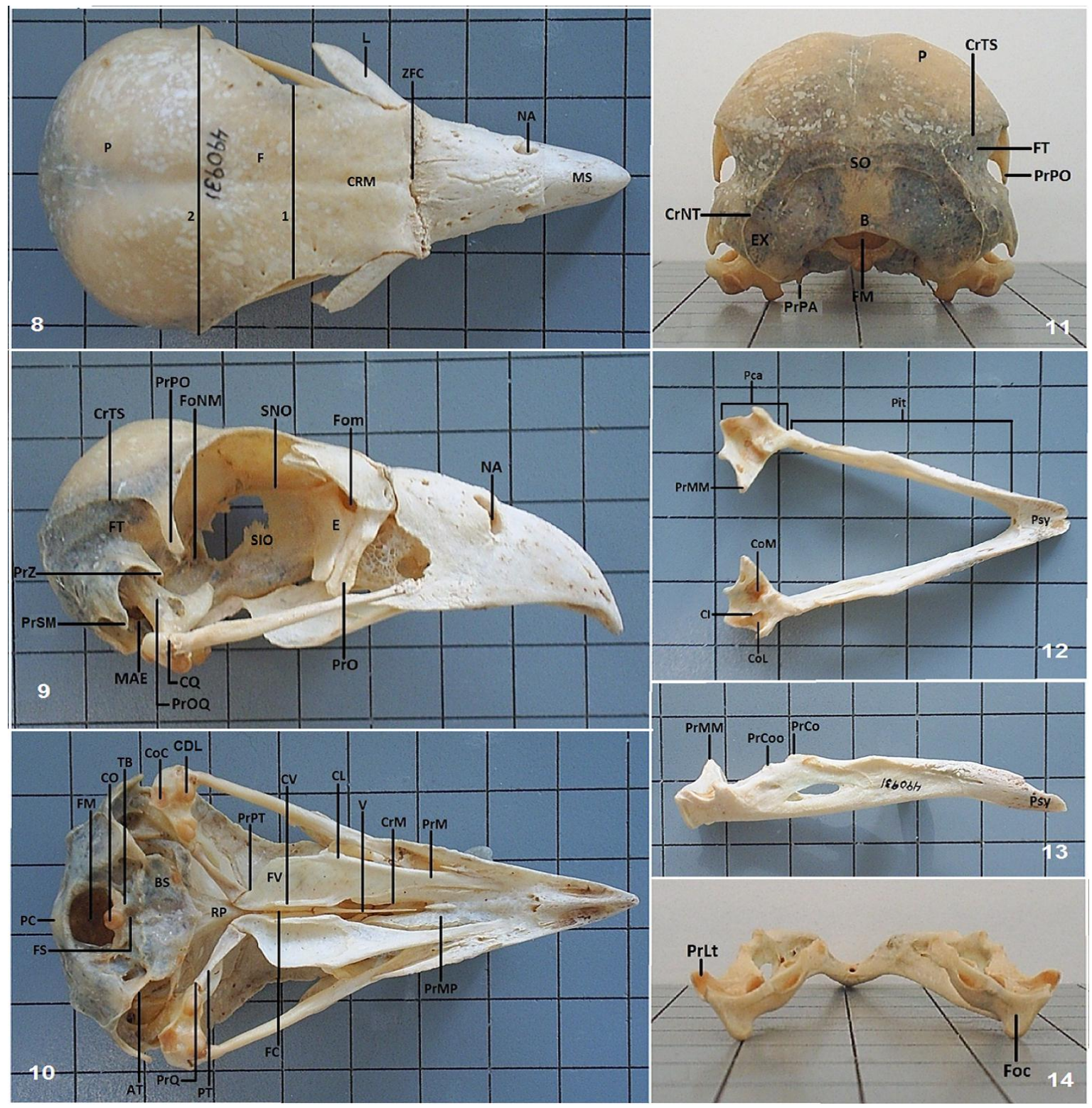

Figuras 8 a 14. Vistas do crânio e mandíbula de Caracara plancus. Notar que o quadriculado da figura tem um centímetro de lado. 8: Vista dorsal do crânio. 9: Vista Lateral do crânio. 10: Vista ventral do crânio. 11:Vista caudal do crânio. 12: Vista dorsal da mandíbula. 13. Vista lateral da mandíbula. 14. Vista caudal dos ramos mandibulares. Legenda: 1: largura interorbital; 2: largura parietal; AT: ala timpânica; B: basioccipital; BS: região basisfenóide; CI: crista intercotilar; CL: crista lateral palatina; CO: côndilo occipital; CoC: côndilo caudal; CDL: côndilo lateral; CoL: cótila lateral; CoM: cótila medial; CQ: corpo do osso quardrado; CrM: cristas mediais palatinas; CRM: concavidade rostral mediana; CrNT: crista nuchalis transversa; CrTS: crista temporal superior; CV:crista ventral palatina; E: ectetmóide; EX: exoccipital; F: frontal; FC: fossa coanal; FM: forâmen magno; Foc: fossa caudal; Fom: forâmen orbitonasal medial; FoNM: forâmen do nervo maxilomandibular; FS: fossa subcondilar; FT: fossa temporal; FV: fossa ventral palatina; L: lacrimal; MAE: meato acústico externo; MS: maxila superior; NA: abertura nasal; P: parietal; PC: Proeminência cerebelar; Pca: parte caudal; Pit: parte intermediária; PrCo: o processo coronóide 1; PrCoo: processo coronóide 2; PrLt: processo lateral da mandíbula; PrM: processo maxilar do palatino; PrMM: processo medial da mandíbula; PrMP: processo maxilopalatino; PrO: processo orbital do lacrimal; PrOQ: processo ótico do quadrado; PrPA: processo paraoccipital; PrPO: processo pós-orbital; PrPT: processo transpalatino; PrQ: processo quadrático do pterigoide; PrSM: processo suprameático; PrZ: processo zigomático; Psy: parte sinfisial da mandíbula; PT: pterigoide; RP: rostro paraesfenoidal; SIO: septo interorbital; SNO: sulco olfatório; SO: supraoccipital; TB: tubérculo basilar; V: vômer; ZFC: zona flexória craniofacial. 
comprimento total da mandíbula nas espécies analisadas. A parte intermediária (pars intermedia- Pit, Figuras 5 e 12) possui uma fenestra mandibular bem desenvolvida, porém rasa em toda a sua extensão, e a parte caudal (pars caudalis -Pca, Figuras 5 e 12) abriga a musculatura responsável pela movimentação das maxilas, além de ser a área de articulação com o osso quadrado.

Na região dorsal da mandíbula observa-se o bem desenvolvido processo coronóide 1 (Proc. coronoideus PrCo, Figuras 6, 7 e 13) em ambas as espécies estudadas, na região do osso supra-angular (Os supra-angulare), na altura da porção caudal da fenestra mandibular. Dorsorostralmente, destaca-se o processo coronóide 2 (PrCoo - Figuras 6, 7 e 13). O processo coronóide 2 é desenvolvido nas espécies analisadas.

Na região dorsocaudal da mandíbula pode ser observada a crista intercotilar (CI - Figuras 5 e 12), pronunciada. A cótila lateral (Cotyla lateralis - CoL, Figuras 5 e 12) e a cótila medial (Cotyla medialis - CoM, Figuras 5 e 12) mostram-se conspícuas. O processo medial (Proc. medialis mandibulae - PrMM, Figuras 5, 7 e 12) apresenta-se bem desenvolvido e projeta-se dorsomedialmente, sendo largo em sua base afilando-se distalmente.

Na mandíbula está presente o bem desenvolvido processo lateral da mandíbula (PrLt, Figuras 5-7 e 14), disposto caudalmente na sua parte posterior. O tubérculo pseudotemporal da região medial da mandíbula (Tuberculum pseudotemporale) é inconspícuo em C. cheriway e em C. plancus.

Na região caudal destaca-se a fossa caudal (Fossa caudalis, Foc, Figura 14), bem desenvolvida, sendo rasa e larga; delimita-se lateralmente com a crista lateral, medialmente com a crista medial e dorsalmente com a crista transversa fossae. O processo retroarticular (Proc. retroarticularis) não foi observado nas espécies estudadas.

\section{Discussão}

A comparação da osteologia craniana de Caracara cheriway e C. plancus revela alguns caracteres compartilhados pelas duas espécies, tais como: um longo processo pós-orbital; a presença do processo suprameático; uma profunda fossa temporal; na região do osso paraesfenóide, destacam-se a projeção do rostro paraesfenoidal, que alcança $50 \%$ da distância do côndilo occipital ao pterigóide; um bem desenvolvido osso ectetmóide; a presença do processo do ectetmóide; o longo processo maxilar do palatino; a longa crista lateral palatina; ausência da projeção nas extremidades caudais do palatino (ou processos transpalatino de alguns autores); a presença de uma profunda e curta fossa ventral palatina; o curto processo pterigóide do palatino; as cristas ventrais palatinas são longas e proeminentes; o vômer apresenta-se como uma lâmina longa e estreita; ausência do processo dorsal do pterigoide; o curto processo orbital do quadrado; o processo ótico do quadrado é longo e alargado; a presença do processo ótico externo e interno do quadrado; o côndilo caudal é maior que o medial; a presença de uma conspícua crista temporal superior; a proeminência cerebelar da região do osso supra-occipital é saliente e afilada; a maxila superior tem cerca de 4/9 do comprimento total do crânio; a parte sinfisial da mandíbula ocupa cerca de $1 / 7$ de seu comprimento total; os processos coronóide 1 e 2 são bem desenvolvidos; o tubérculo pseudotemporal da região medial da mandíbula é inconspícuo; a ausência do processo retroarticular; a parte proximal do lacrimal termina de forma arredondada e a parte proximal do lacrimal tem cerca de 1/4 da largura da parte distal. A manutenção do compartilhamento dessas características pode indicar pouco tempo de divergência entre essas duas espécies, pois, segundo FUCHS et al. (2012), elas diferem entre si somente em $0,5 \%$ quanto aos genes mitocondriais.

O estreitamento lateral do osso frontal na região das órbitas nas aves é associado à ampliação do campo visual (BURTON, 1984) e há uma diferença no comportamento reprodutivo que pode estar relacionada ao maior estreitamento interorbital em Caracara cheriway. Nessa espécie há intensa competição por território (ocupação delimitada pela densidade), e o aprimoramento social depende do contato visual entre os indivíduos, que ocupam preferencialmente áreas abertas (DWYER et al., 2013). A identificação visual dos grupos formados por jovens oportunistas, por parte dos casais fieis ao seu território, pode ser favorecida por sua maior amplitude visual, ao passo que em C. plancus as interações hierárquicas intra-específicas são mais rígidas, e as aves imaturas são deslocadas pelas adultas reprodutoras para áreas menos atrativas (TRAVAINI et al., 2001), como destacado por BAUMGARTEN (1998), que observou um expressivo aumento no número de jovens de C. plancus no Parque Nacional das Emas (GO) nos meses de outubro a novembro (época que coincide com o final do período reprodutivo) e depois uma rápida diminuição desta proporção, indicando uma massiva saída dos jovens do parque (BAUMGARTEN, 1998). Em contrapartida, os juvenis de $C$. cheriway formam grupos e utilizam as áreas de forrageamento de seus pais o máximo de tempo possível até delimitarem seus próprios territórios (DWYER et al., 2013).

O processo pós-orbital é longo em $C$. cheriway e $C$. plancus, ao passo que em muitas espécies de Falconidae, como Mivalgo gilvicollis, M. ruficollis (GUZZI et al., 2014) e M. semitorquatus (SILVA et al., 2012) é mais curto e robusto. O processo pós-orbital delimita anteriormente a fossa temporal, que é profunda, o que indica que o músculo adutor mandibular é bem desenvolvido, o que pode estar relacionado à dilaceração das presas durante o processo de alimentação. $\mathrm{O}$ músculo adutor mandibular e o grupo de músculos pseudotemporais são os principais responsáveis pela prevenção da desarticulação da mandíbula, puxando-a para trás (SUSTAIA, 2008). Aliado a isso, a região esquamosal projeta-se ros- 
tralmente e forma o processo zigomático. Tal processo apresenta-se longo e alargado em sua base e tem forma de um triângulo característico, levemente torcido sobre si mesmo, ocupando cerca de $40 \%$ da distância entre a sua origem no crânio e a barra jugal em C. cheriway, em 35\% em C. plancus.

A região frontal continua-se caudalmente com a ampla região parietal e laterocaudalmente com o curto processo pós-orbital. Em virtude da fusão da maior parte dos ossos do crânio nas Aves, há dificuldade para estabelecer seus limites, bem como suas respectivas participações na formação de determinadas estruturas associadas (BAUMEL; WITMER, 1993). O aumento da massa corporal dos Falconidae está diretamente relacionado ao aumento do crânio, que por sua vez permite uma maior inserção dos músculos do pescoço ( $M$. rectus capitis lateralis e M. splenius capitus), que lhes confere maior vantagem mecânica na alimentação (SUSTAITA, 2008). Dentre as aves de rapina, os carniceiros possuem um crânio mais estreito quando comparado aos avívores como as espécies que compõem Micrastur, mas a distância entre a crista opistótica e a crista occipital são comparativamente maiores que nos demais raptores, e a posição do forame magno é mais posterior ao crânio, o que permite uma inserção mais linear da coluna vertebral, e quando somadas, essas características facilitam os movimentos laterais e aumentam a força para que essas aves consigam desprender a carne da carcaça (HERTEL, 1995; SUSTAITA; HERTEL, 2010). Esse comportamento generalista/carniceiro surge nos Caracarinae entre $14 \mathrm{e}$ 6.7 M.a., e essa diversificação comportamental pode estar ligada à aridização do clima na região Neotropical, que acompanhou a expansão das áreas abertas e o aumento da abundância de mamíferos herbívoros, potencial fonte de alimento (FUCHS et al., 2012).

A razão entre a distância da parte distal do lacrimal ao arco da órbita e a distância do arco da órbita até a barra jugal e a razão entre a distância das partes terminais dos dois lacrimais e o diâmetro parietal são diferentes nas duas espécies de Caracara, o que confere um lacrimal mais destacado da órbita, e mais alto a $C$. cheriway. CRACRAFT (1968) discutiu a função do complexo lacrimal-ectetmóide para as aves, relacionando o desenvolvimento de tais estruturas às pressões seletivas que atuam sobre tais processos, particularmente relacionado aos hábitos alimentares das espécies. As duas subfamílias de Falconidae possuem estratégias de caça bem diversa. Enquanto os Falconinae (Falco entre outros) são avívores, ou seja, predam outras aves em pleno voo, matando suas presas com suas garras e bico, os Caracarinae possuem diversas estratégias de caça: Daptrius e Ibicter são onívoros e arbóreos, enquanto Milvago, Caracara, e Phalcoboenus, alimentam-se de uma dieta geralista, como invertebrados, vertebrados e carniça (FUCHS et al., 2012). Estes últimos três gêneros são em grande parte terrícolas, com características morfológicas distintas, com pernas relativamente longas e dedos curtos (MOSTO et al., 2013). Aliado a isso, C. cheriway também se alimenta de nozes, no Texas central (SKORUPPA; LEE, 2008) e C. plancus dispersa frutos de bacuri (Attalea phalerata) no Pantanal (GALETTI; GUIMARÃES, 2004).

O processo orbital do osso quadrado é curto e o processo ótico do quadrado é longo e alargado nas duas espécies de Caracara. Embora ainda haja discordâncias sobre os movimentos exatos do osso quadrado durante a abertura do bico, praticamente todos os autores concordam no que diz respeito à função desempenhada pelo osso quadrado durante a cinese craniana: ele é o ponto inicial a partir do qual se originam todos os movimentos cinéticos cranianos (BOCK, 1964; BÜHLER, 1981; ZUSI, 1984; BURTON, 1984), que transmitem ao palatino, através do pterigoide, força e direcionamento para a maxila superior. Sua forma e espessura estão associadas também ao tamanho e à força da maxila superior, bem como à resistência da musculatura (BOCK, 1964, 1966). $\mathrm{O}$ quadrado aliado ao pterigoide e ao $m$. pterigoideus são as principais estruturas relacionadas à transmissão de força ao "dente tomial" (falso dente no bico), que é empregado pelos Falconidae em um movimento antero -posterior das maxilas para matar e desossar suas presas (SUSTAITA; HERTEL, 2010).

A sínfise mandibular das espécies de Caracara é menor do que as das duas outras espécies estudadas, e aliado à inserção da musculatura mandibular, a espessura dos ramos mandibulares e o comprimento da sínfise mandibular constituem as principais variáveis relacionadas à força da mordida, pois quanto maior a distância entre a inserção dos músculos mandibulares e o eixo de rotação mandibular, quanto mais largo os ramos mandibulares e quanto maior a sínfise mandibular, maior será a força da mordida e, consequentemente, maior será o sucesso na predação, pois os membros de Falconidae, embora capturem as presas com as garras, matam essas presas com o bico, quebrando sua coluna vertebral com uma força $64 \%(2.7 \mathrm{~N}$ a $24.5 \mathrm{~N})$ superior à empregada pelos Accipitridae (1.4 N a 5.4 N) (SUSTAITA, 2008; SUSTAITA; HERTEL, 2010). Os Accipitridae, Pandionidae e Vulturidae, quando comparados aos Falconidae, possuem a mandíbula mais longa e a sínfise mandibular maior, o que lhes permitem explorar uma maior gama de itens alimentares, como mamíferos, répteis, peixes e até mesmo carcaças em decomposição (HERTEL, 1995).

A variação observada na anatomia craniana entre M. gilvicollis, M. ruficollis (GUZZI et al., 2014) e M. semitorquatus (SILVA et al., 2012) mostra alguns caracteres exclusivos do gênero Caracara, tais como: a parte proximal do lacrimal tem cerca de $1 / 4$ da largura da parte distal; apars symphisialis mandibulae ocupa cerca de 1/7 do comprimento total da mandíbula; a maxila superior tem cerca de 4/9 do comprimento total do crânio; a parte sinfisial da mandíbula ocupa cerca de $1 / 7$ de seu comprimento total. Dentre as características osteológicas analisadas, os caracteres exclusivos para C. cheriway, quando comparado a C. plancus, M. gilvicollis, M. ruficollis 
(GUZZI et al., 2014) e M. semitorquatus (SILVA et al., 2012) são: a largura interorbital é cerca de $1 / 2$ daquela da região parietal; na região da zona flexória craniofacial apresenta uma proeminência do frontal; a distância entre as partes distais dos dois lacrimais atinge 5/6 da largura parietal; a razão entre o diâmetro interorbital e a região parietal varia de 2 vezes; o processo zigomático ocupa cerca de $40 \%$ da distância entre a sua origem no crânio e a barra jugal. C. plancus, quando comparado a C. cheriway, M. gilvicollis, M. ruficollis (GUZZI et al., 2014) e M. semitorquatus (SILVA et al., 2012) possui a largura interorbital cerca de 4/7 da largura parietal; na região da zona flexória craniofacial há uma concavidade rostral mediana; a distância entre as partes distais dos dois lacrimais atinge 6/7 da largura parietal; a razão entre o diâmetro interorbital e a região parietal varia de 1,75 vezes; o processo zigomático ocupa cerca de 35\% da distância entre a sua origem no crânio e a barra jugal.

Diversas características do comportamento alimentar parecem ter selecionado algumas adaptações no crânio das espécies de Cacaraca, quando comparadas a outras espécies de Falconidae, e a maioria delas está relacionada ao hábito carniceiro, oportunista e generalista, e a diferença no comportamento reprodutivo pode ter selecionado as diferenças osteológicas cranianas entre essas duas espécies. Desta forma foi possível, através da osteologia craniana comparada, levantar caracteres compartilhados pelas espécies do gênero Caracara, e exclusivas de cada uma das duas espécies que o compõem, indicando que a morfologia, a despeito de outros métodos de inferência filogenética, ainda pode prover caracteres úteis à sistemática.

\section{Agradecimentos}

Os autores são gratos aos curadores da coleção osteológica da Divisão de Aves do Smithsonian Institution, National Museum of Natural History (USNM) por permitir o amplo acesso aos espécimes descritos nesse estudo. Agradecem também aos dois revisores anônimos pela cuidadosa leitura do manuscrito, e pelas sugestões pertinentes.

\section{Referências}

ACTKINSON, M. A.; KUVLESKY JUNIOR., W. P.; BOAL, C. W.; BRENNAN, L. A.; HERNANDEZ, F. Nesting habitat relationships of sympatric Crested Caracaras, Red-Tailed Hawks, and White-Tailed Hawks in South Texas. The Wilson Journal of Ornithology, Oberlin, v. 119, n. 4, p. $570-578,2007$.

BAUMEL, J.; WITMER, L.M. Osteology. In: Baumel, J., King, A.S., Breazile, J.E., Evans, H.E., Vander Berge, J.C. (eds). Handbook of Avian Anatomy:
Nomina Anatomica Avium. 2. ed. Cambridge: Nutall Ornithologists Club. 1993. p. 45-132.

BAUMGARTEN, L. C. Ecologia dos Falconiformes de áreas abertas no Parque Nacional das Emas (Mineiros/ GO). Dissertação (Mestrado) - Universidade Estadual de Campinas, p. 1 - 80, 1998.

BLAKE, E. R. Manual of neotropical Birds. Vol. 1 Sphenicidae (Pinguins) to Ralidae (Gulls e Allies). The University of Chicago Press, Chicago e London. 1977. 724p.

BOCK, W. J. Kinetics of the avian skull. Journal of the Morphology, Philadelphia, 114, 1-41, 1964.

BROWN, L.; AMADON, D. Eagles, hawks and falcons of the world. New York: McGraw-Hill Book Co., 1968. 531p.

BÜHLER, P. Functional anatomy of the avian jaw apparatus, p. 439-468. In: A. S. King \& J. McLelland (Eds). Form and Functions in Birds. London, Academic Press, vol. 2, 1981. p. 496.

BURTON, P. J. K. Anatomy and evolution of the feeding apparatus in the avian orders Coraciiformes and Piciformes. Bulletin of the British Museum (Natural History), London, 47, 6, 1984, 331- 443.

COMITÊBRASILEIRODE REGISTROSORNITOLÓGICOS (CBRO) 2014. Lista de Aves do Brasil Versão 2014. Disponível em: http//www.crbo.org.br Acessado em: Março 10, 2014.

CRACRAFT, J. The Lacrimal- ectehmoid bone complex in bird: a single character analysis. American Midland Naturalist, v. 80, n. 2, p. 316-359, 1968

Del HOYO, J.; ELLIOTT, A.; SARGATAL, J. Handbook of the birds of the world. v. 2. Barcelona: Lynx Edicions, 1994.

DONATELLI, R. J. Cranial osteology and miology of the jaw apparatus in the Galbulidae (Aves, Piciformes). Arq. Zool., São Paulo, v. 32, n. 1, p. 32, 1992.

DOVE, C.J.; R.C. BANKS.A taxonomic study of crested caracaras (Falconidae). Wilson Bulletin 111:330-339, 1999.

DWYER, J. F.; FRASER, J. D.; MORRISON, J. L. Range sizes and habitat use of non-breeding Crested Caracaras in Florida. Journal of Field Ornithology, Waco, v. 84, n. 3, p. 223 - 233, 2013.

FERGUSON-LEES, J.; CHRISTIE, D. A. Raptors of the 
world. Houghton and Mifflin Company. Boston, MA, 2001

FRANZO, V. S.; ARTONI, S. M. B.; VULCANI, V. A. S.; SAGULA, A.; MORAES, C. Análise biométrica do intestino do carcará (Polyborus plancus, Miller 1777). Biotemas, Florianópolis, v. 20, n. 2, p. 83-88, 2007.

FRIEDMANN, H. The birds of North and Middle America. Part XI. Bull. 50. Smithsonian Institution, Washington, 1950.

FUCHS, J.; CHEN, S.; JOHNSON, J. A.; MINDELL, D. P. Pliocene diversification within the South American Forest falcons (Falconidae: Micrastur). Elsevier, 60, 398-407, 2011.

FUCHS, J.; JOHNSON, J. A.; MINDELL, D. P. Molecular systematics of the caracaras and allies (Falconidae: Polyborinae) inferred from mitochondrial and nuclear sequence data. Ibis, Oxfordshire, v. 154, n. 3, p. 520 $-532,2012$.

GALETTI, M.; GUIMARÃES JUNIOR, P. G. Seed dispersal of Attalea phalerata (Palmae) by Crested caracaras (Caracara plancus) in the Pantanal and a review of frugivory by raptors. Ararajuba, Pará, v. 12, n. 2, p. 133-135, 2004.

GILL, F.; DONSKER, D.. 2014. IOC word bird names (version 4.1) Disponível em http://www. worldbirdnames.org/ [Acessado "22/03/2014"].

GLOBAL RAPTORS Information Network. 2010. Species account: Crested Caracara Caracara cheriway. Downloaded from http://www.globalraptors.org on 27 Feb. 2010

GRIFFITHS, C. S. Phylogeny of the falconidae inferred from molecular and morphological data. The Auk, Oakland, v. 116, n. 1, p. 116 - 130, 1999.

GRIFFITHS, C. S; BARROWCLOUGH, G. F.; GROTH, G.; MERTZ, L. Phylogeny of the Falconidae (Aves): a comparison of the efficacy of morphological, mitochondrial, and nuclear data. Molecular Phylogenetics and Evolution, Philadelphia, v.32, n. 101-109, 2004.

GUZZI, A.; SANTOS, F. C. V.; FERREIRA, G. J. B. C.; DONATELLI, R. J. Comparative analysis of the cranial osteology and evolutionary traits of Micrastur gilvicollis, Micrastur ruficollis and Micrastur semitorquatus (Aves: Falconiformes). Revista Nordestina de Zoologia, Recife, v. 8, n. 1, p. 70 - 88, 2014.

HELLMAYIR, C.E.; CONOVER, B. Catalogue of birds of the Americas, part 1. Chicago, Field Museum of Natural History, v.13, n.4, 1949, 359p.

HERTEL, F. Ecomorphological indicators of feeding behavior in recent and fossil raptors. Auk, Oakland, v. 112, n. 4, p. 890-903, 1995.

HOFLING, E. Aves no Campus. São Paulo. Instituto de Biociências da Universidade de São Paulo, 126p., 1993.

LIVEZEY, B.C. A phylogenetic analysis of recent anseriformes genera using morphological characters. Auk, v.103, p.737-754, 1986.

LIVEZEY, B.C. A phylogenetic analysis and classification of Recent dabbling ducks (Tibe Anatini) based on comparative morphology. Auk, v.108, p.471-507, 1991.

LIVEZEY, B.C.; ZUSI, R.L. Higher-order phylogenetics of modern Aves based on comparative anatomy. II Analysis and discussion. Zoological Journal of the Linnean Society, V.149, p. 1-95, 2007.

MONTALVO, C. I.; TALLADE, P. O.; FERNÁNDEZ, F. J.; MOREIRA, G. J.; RAFUSE, D. J.; De SANTIS, L. J. M. Bone damage patterns found in the avian prey remains of crested caracara Caracara plancus (Aves, Falconiformes). Journal of Archaeological Science, Amsterdam, v. 38, n. 12, p. 3541-3548, 2011.

MORRISON, J. L. Age-specific survival of Florida's Crested Caracaras. Journal of Field Ornithology, Waco, v. 74, n. 4, p. 321-330, 2003.

MORRISON, J. L.; ABRAMS, J.; DEYRUP, M.; EISNER, T.; MCMILLIAN, M. Noxious Menu: Chemically Protected Insects in the Diet of Caracara cheriway (Northern Crested Caracara). Southeastern Naturalist, Steuben, v. 6, n. 1, p. 1 - 14, 2007.

MORRISON, J.L.; PIAS, K.E.; ABRAMS, J.; GOTTLIEB, I.G.W.; DEYRUP, M., AndMcMILLIAN, M. Invertebrate diet of breeding and nonbreeding caracaras (Caracara cheriway) in Florida. Journal of Raptor Research, v.12, p.38-47, 2008.

MOSTO, M. C.; CARRIL, J.; PICASSO, M. B. J. The Hindlimb Myology of Milvago chimango (Polyborinae, Falconidae). Journal of Morphology, New Jersey, v. 274, n. 10, p. 1191- 1201, 2013.

NEGRO, J. J.; SARASOLA, J. H.; FARIÑAS, F.; ZORRILLA, I. Function and occurrence of facial flushing in birds. Comparative Biochemistry and Physiology, Philadelphia, v. 143, n. 1, p. $78-84,2006$.

POSSO, S. R.; DONATELLI, R. J. Cranial osteology and 
systematic considerations in Coccyzinae (Cuculidae). Bol. Mus. Para. Emílio Goeldi. Ciências Naturais, Belém, v. 2, n. 1, p. 87-116, jan-abr. 2007.

RIDGWAY, R. Studies of the American Falconidae. Monographof the Polybori. Bull. U. S. Geol. Geogr. Surv. Territories 1, 451-473, 1876

SAZIMA, I. The jack-of-all-trades raptor: versatile foraging and wide trophic role of the Southern Caracara (Caracara plancus) in Brazil, with comments on feeding habits of the Caracarini, Revista Brasileira de Ornitologia, Pará, v. 15, n. 4, p. 592-59, 2007.

SIBLEY, C. G.; MOROE, B. L. Distribution and taxonomy of birds of the word. New Haven e London: Yale University Press, 1990. 1111p.

SICK, H. Ornitologia brasileira. 3. ed. :Rio de Janeiro: Nova Fronteira, Parte 2, cap.10, p. 233-243, 1997.

SILVA, A. G.; G. J. B. FERREIRA; R. J. DONATELLI, A. GUZZI. Cranial osteology of Micrastur semitorquatus Vieillot, 1817 (Falconiformes: Falconidae). Comunicata Scientiae, Bom Jesus, v. 3, n. 1, p. 64-71, 2012.

SKORUPPA, M. K.; LEE, M. C. Crested Caracaras Feed on Pecans in South-central Texas. Journal of Raptor Research, Westchester, v. 42, n. 4, p. 299 - 300, 2008.

STRESEMANN, E.; AMADON, D. Falconiformes. In: Mayr, E. and Cottrell, G. W. (eds). Check-list of the birds of the world, vol. 1, 2nd ed. Harvard Univ. Press, Cambridge, 1979, p. 271- 424.

SUSTAITA, D. Musculoskeletal underpinnings to differences in killing behavior between North American accipiters (Falconiformes: Accipitridae) and falcons (Falconidae). Journal of Morphology, New Jersey, v. 269, n. 3, p. 283 - 301, 2008.

SUSTAITA, D.; F. HERTEL. In vivo bite grip forces, morphology and prey-killing behavior of North American accipiters (Accipitridae) and falcons (Falconidae). The Journal of Experimental Biology, Cambridge, v. 213, p. 2617-2628, 2010.

TRAVAINI, A.; DONAHZAR, J. A.; CEBALLOS, O.; HIRALDO, F. Food habits of the Crested Caracara (Caracara plancus) in the Andean Patagonia: the role of breeding constraints. Journal of Arid Environments, Amsterdã, v. 48, p. 211 - 219, 2001.

VITALIANO, S. N.; MINEO, T. W. P.; ANDRÉ, M. R.; MACHADO, R. Z.; MINEO, J. R. WERTHER, K. Experimental infection of Crested Caracara (Caracara plancus) with Toxoplasma gondii simulating natural conditions. Veterinary Parasitology, Amsterdã, v. 172, n. 1-2, p. 71 - 75, 2010.

VUILLEUMIER, F. Generic relations and speciation patterns in the Caracaras (Aves: Falconidae). Brevoria, Cambridge, n. 355, p. 1 - 29, 1970.

WETMORE, A. 1965. The birds of the Republic of Panama. Part I. Smithsonian Miscellaneous Collection, v. 150. $483 p$.

ZOTTA, A. R. Lista sobre el contenido estomacal de las aves argentinas. Hornero, Buenos Aires, v. 7, n. 3, p. 402 - 411, 1940.

ZUSI, R. L. A functional and evolutionary analysis of rhynchokinesis in birds. Smithson. Contrib. Zool., n. 395, p. 1-40, 1984.

ZUSI, R.L. Patterns of diversity in the avian skull. In: Haken, J.; Hall, B.K. eds. The skull. Chicago, Chicago University Press. v. 2, p. 391-437, 1993. 